\title{
Central mechanisms of obesity and related metabolic diseases
}

\author{
Dongsheng Cai
}

Published online: 22 October 2013

(C) Springer Science+Business Media New York 2013

Obesity and associated metabolic syndrome are growing global problems which have taken an epidemic stature over recent decades. According to International Obesity Task Force and World Health Organization's guidelines, approximately 1.0 billion adults are currently overweight with Body Mass Index (BMI) of 25-29.9 kg/m², and 475 million are obese (BMI greater than or equal to $30 \mathrm{~kg} / \mathrm{m}^{2}$ ). Prevalence of threats from these disorders poses a major risk for many deleterious, chronic diseases, such as type-2 diabetes (T2D), stroke and coronary heart disease and cancers, thus exerting a heavy toll on healthcare economy and demanding a big attention from clinicians, researchers and policy makers.

Through recent research, considerable advances have been made towards therapeutic approaches for obesity and related metabolic disorders, for example, a few newer drugs with better pharmacological activities, and gastric surgery for treating severe obesity. However, obesity tide continues to escalate in particular in developed countries. Thus, the challenge to preventive and therapeutic success remains, partly due to the fact that obesity pathophysiology is orchestrated by complex interactions among environment, genetic predisposition and behaviors. With this pressing experimental and clinical need, extensive research in this direction is ongoing, be it in the field of gaining mechanistic insights into neuroendocrine regulation of body weight, or targeting inhibition of peripheral and central anabolic factors or stimulation of catabolic signals. Earlier research has linked obesity to a chronic imbalance between energy intake and expenditure, that results in inordinate lipid depositions in adipose tissues which activate various stress responsive and inflammatory pathways. Recent research developments have

D. Cai $(\bowtie)$

Department of Molecular Pharmacology, Albert Einstein College of Medicine, 1300 Morris Park Avenue, Bronx, NY 10461, USA

e-mail: dongsheng.cai@einstein.yu.edu linked defects of hypothalamic signaling to obesity and metabolic syndrome which is considerably expanding the scope of the mechanistic understanding.

This special edition in Reviews in Endocrine and Metabolic Disorders highlights a series of recent advancements in understanding the brain regulation of metabolic disorders and potential new therapeutic options. Blevins and Ho provide an excellent overview about the implications of oxytocin signaling disruption in obesity manifestation and the potential of peripheral or central oxytocin treatment as a weight loss therapeutic target. Considering oxytocin treatment has shown promising results in a recent clinical trial, further research in experimental and clinical levels is much needed for developing a safe and effective treatment option. M. H. Tschöp and his colleagues focus on an important but often neglected subject of sexual dimorphism and role of gonadal hormones in obesity and metabolic diseases. They deliberated on astrocytic estrogen-mediated neuroendocrine regulation of body weight and metabolic homeostasis. He and Feng discussed the role of crosstalk between estrogen and leptin signaling in metabolic balance, a very relevant and timely topic of active research in the light of weight management and breast cancer development. Seli and Horvath delve upon this important topic describing how vaginal birth in advantage to experimental C-section induces higher hippocampal Ucp2 mRNA expression thus enabling metabolic adaptation to available nutrients and regulation of postnatal neuronal differentiation, axonal outgrowth, synapse formation and adult behaviors. The importance of adult neuronal modifications has been further highlighted by Purkayastha and Cai while discussing an obesogenic effect of prolong high-fat diet-induced hypothalamic inflammation via disruption of hypothalamic neurogenesis and neural stem cells. Butterick et al. discussed if hypothalamic neuropeptide orexin A may promote obesity resistance by increasing spontaneous physical activity. T. K. Lam and his colleagues discussed on how two pancreatic hormones, insulin and glucagon, could work in 
consonance in the brain for the regulation of energy and glucose homeostasis. M. S. Lee and group discussed the prospect of hypothalamic autophagy on becoming a novel therapeutic target for appetite control and whole-body metabolism. L. Rui has given a comprehensive overview of the homeostatic and hedonic circuits of the hypothalamus and the hind brain that regulate food intake and energy expenditure, as well as potential neural defects that contribute to obesity pathogenesis.

These in-depth reviews provide a wealth of information and can expand readers' understandings of the cellular and mechanistic bases of obesity and related disorders while illuminating the current therapeutic options and potential drug targets. Additionally, many of these reviews provide angles to promote research interest to address the lingering obstacles of effective and safe therapeutic options for long-term weight loss and reducing metabolic disorders. It is our hope that continuing research will lead up to novel therapeutic strategies for this complex cluster of difficult-to-treat metabolic disorders and diseases.

As Guest Editor of this issue, I would take this opportunity to thank the contributing authors who have enlightened us on some very important and timely topics and newer approaches. My sincere gratitude is to the editorial and production staff of Springer for patiently accommodating all our contributions.

Conflict of interest The author declares that there is no conflict of interest. 